\title{
Multi-analyte Biosensor Chip for Determination of Organic Acids in Biogas Processes
}

\author{
Johanna Pilas $^{1,2}$, D.L. Röhlen ${ }^{1}$, Thorsten Selmer ${ }^{1}$, Michael Keusgen ${ }^{2}$, Michael J. Schöning ${ }^{1,3}$ \\ 1 Institute of Nano- and Biotechnologies, FH Aachen, Heinrich-Mußmann-Str. 1, Jülich, Germany, \\ ${ }^{2}$ Department of Pharmaceutical Chemistry, Philipps-Universität Marburg, Marbacher Weg 6-10, 35037 \\ Marburg, Germany, \\ ${ }^{1}$ Institute of Complex Systems, Forschungszentrum Jülich GmbH, Wilhelm-Johnen-Str., Jülich, \\ Germany \\ pilas@fh-aachen.de
}

\begin{abstract}
:
This contribution describes the development and characterization of a multi-analyte biosensor system for simultaneous detection of up to four different analytes. The bi-enzymatic detection principle enables facile determination of ethanol, formate, D- and L-lactate. For immobilization of the required enzymes, a chemical cross-linking step with glutaraldehyde was applied. Combination of various analyte-specific electrodes, within one biosensor array, provides a broader insight into the complex nature of fermentation processes. Application of the biosensor chip for detection of organic acids in real samples, such as silage (maize and sugar cane) and fermentation sludge from a biogas plant, is presented. Comparison with a conventional reference technique demonstrates a promising potential of the multi-analyte biosensor for rapid monitoring of complex media.
\end{abstract}

Key words: biogas, biosensor, enzyme, multi-analyte, organic acids

\section{Introduction}

Monitoring of complex fermentation processes, like the methane production, is crucial for a stable and efficient conversion of assigned substrate to the desired product [1]. In this regard, determination of several key parameters is advantageous for improved evaluation of the current status. Especially, organic acids and volatile fatty acids are important indicators for process imbalances during methane production [2,3]. These compounds are typically analyzed by conventional methods, like high pressure liquid chromatography, gas chromatography or spectrophotometry. However, these techniques are rather time-consuming and require laborious sample preparation. Facile and fast detection systems are therefore desirable devices for the on-site monitoring of fermentation samples.

\section{Chip Design and Sensor Principle}

The biosensor chip $\left(14 \times 14 \mathrm{~mm}^{2}\right)$ is fabricated in conventional thin-film technology and comprises five individual working electrodes and an integrated common counter electrode. Fig. 1 shows an image of the developed biosensor. Each platinum electrode $(\varnothing 2 \mathrm{~mm}$ each) is modified with an analyte-specific $\mathrm{NAD}^{+}$-dependent dehydrogenase and diaphorase from Clostriudium kluyveri [4]. In this way, an electrochemical detection principle is realized for simultaneous analysis of different analytes (ethanol, formate, D- and L-lactate) at the same applied working potential of $+300 \mathrm{mV}$ vs. $\mathrm{Ag} / \mathrm{AgCl}$ reference electrode. At this potential the enzymatically produced ferrocyanide (product of diaphorase reaction) is oxidized at the sensor surface, resulting in an increase of the current signal. For facile application of the multi-analyte biosensor, a portable measurement set-up is developed, too.

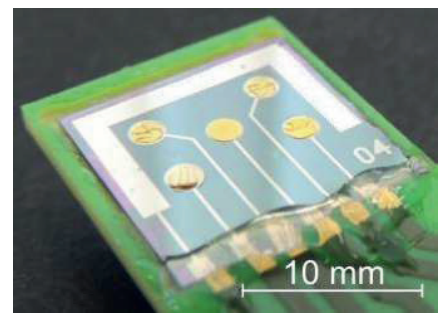

Fig. 1. Photograph of multi-analyte biosensor chip $\left(14 \times 14 \mathrm{~mm}^{2}\right)$ with five circular working electrodes ( $\varnothing$ each $2 \mathrm{~mm}$ ) and an integrated counter electrode. 


\section{Experimental}

Application of the biosensor was performed in silage (maize and sugarcane) and fermentation sludge from a lab-scale biogas plant. Fig. 2a shows exemplarily an amperometric measurement for determination of ethanol, formate, D- and L-lactate in maize silage (typical feedstock for methane production). A working electrode without catalytic activity, functionalized only with bovine serum albumin (BSA), served as a blank signal. With addition

a

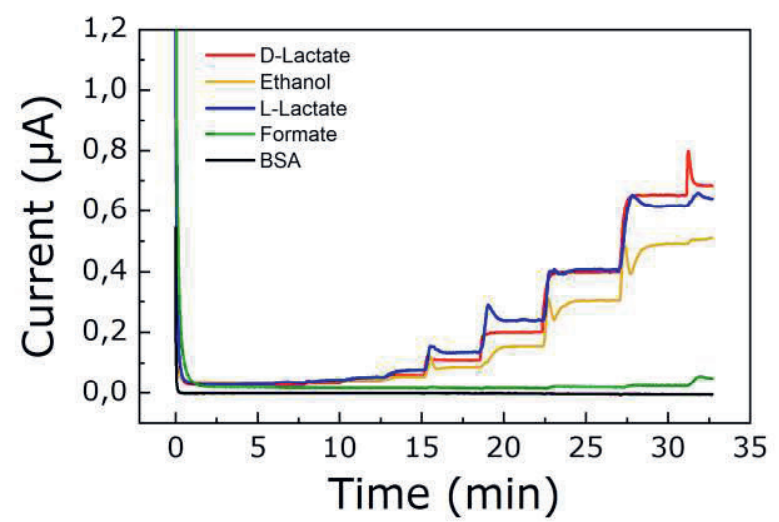

of different analytes (ethanol, formate, D- and Llactate, respectively), the signal of the BSA electrode remains constant, demonstrating no cross-sensitivity to other analytes in the sample. For comparison, reference measurements were conducted with commercial photometric kits. As presented in Fig. 2b, concentrations obtained with the multi-analyte biosensor are in good agreement to the reference technique.

b

Fig. 2. a) Example of an amperometric measurement using the multi-analyte biosensor for organic acid determination in maize silage. b) Comparison of amperometric and photometric (reference) technique for varying concentrations of different organic acids (n.d.: not detectable).

\section{Conclusion}

In summary, this biosensor array can be applied for simultaneous detection of four different organic acids in complex media. The extension of the system with further analytespecific electrodes [5] provides a promising platform for rapid on-site monitoring of various fermentation processes.

\section{Acknowledgements}

The project was supported by the German Federal Ministry of Food and Agriculture (BMEL) (FKZ: 22006613).

\section{References}

[1] L. Janke, A. Leite, M. Nikolausz, T. Schmidt, J. Liebetrau, M. Nelles, W. Stinner, Biogas production from sugarcane waste: assessment on kinetic challenges for process designing, International Journal of Molecular Sciences 16, 20685-20703 (2015); doi:10.3390/ijms160920685

[2] P. Pipyn, W. Verstraete, Lactate and ethanol as intermediates in two-phase anaerobic digestion, Biotechnology and Bioengineering 23, 1145-1154 (1981); doi: 10.1002/bit.260230521

[3] B.K. Ahring, M. Sandberg, I. Angelidaki, Volatile fatty acids as indicators of process imbalance in anaerobic digestors. Applied Microbiology and Biotechnology 43, 559-565; doi: 10.1007/BF00218466
[4] J. Pilas, Y. Yazici, T. Selmer, M. Keusgen, M.J. Schöning, Optimization of an amperometric biosensor array for simultaneous measurement of ethanol, formate, D- and L-lactate, Electrochimica Acta 251, 256-262 (2017); doi: 10.2016/j.electacta.2017.07.119

[5] D.L. Röhlen, J. Pilas, M.J. Schöning, T. Selmer, Applied Biochemistry and Biotechnology 183, 566-581 (2017); doi: 10.1007/s12010-017-2578-1 